\title{
Distribución espacial de mosca mexicana de la fruta (Anastrepha spp.) (Diptera: Tephritidae) en Michoacán, México
}

\author{
Spatial distribution of the Mexican fruit fly complex (Anastrepha spp.) (Diptera: \\ Tephritidae) in Michoacán, Mexico
RICARDO VANEGAS-CARRILLO ${ }^{1}$; JOSÉ FRANCISCO RAMÍREZ-DÁVILA²; ROBERTO RIVERA-MARTÍNEZ ${ }^{3}$

\begin{abstract}
${ }^{1}$ Estudiante de la carrera de Ingeniero Agrónomo Fitotecnista, Facultad de Ciencias Agrícolas, Universidad Autónoma del Estado de México, Campus Universitario “El Cerrillo", El Cerrillo Piedras Blancas s/n, C. P. 50200, Toluca, Estado de México, México, rikiii 03@hotmail.com, https://orcid.org/0000-00027896-0533. ${ }^{2}$ Profesor-Investigador. Responsable del Laboratorio de Entomología de la Facultad de Ciencias Agrícolas, Universidad Autónoma del Estado de México, Campus Universitario “El Cerrillo”, El Cerrillo Piedras Blancas s/n, C. P. 50200, Toluca, Estado de México, México, jframirezd@uaemex.mx, https:// orcid.org/0000-0002-8625-4655. ${ }^{3}$ Doctorado en Ciencias Agropecuarias y Recursos Naturales Facultad de Ciencias Agrícolas, Universidad Autónoma del Estado de México, Campus Universitario “El Cerrillo”, El Cerrillo Piedras Blancas s/n, C. P. 50200, Toluca, Estado de México, México, rob_m@live.com.mx, https://orcid.org/0000-0002-8593-6988.
\end{abstract}

\begin{abstract}
Autor para correspondencia
José Francisco Ramírez-Dávila. Profesor-Investigador. Responsable del Laboratorio de Entomología de la Facultad de Ciencias Agrícolas, Universidad Autónoma del Estado de México, Campus Universitario "El Cerrillo", El Cerrillo Piedras Blancas s/n, C. P. 50200, Toluca, Estado de México, México, jframirezd@uaemex.mx, https://orcid.org/0000-0002-8625-4655.
\end{abstract}

\section{Citación sugerida}

VANEGAS-CARRILLO, R.; RAMÍREZ-DÁ-

VILA, J. F.; RIVERA-MARTÍNEZ, R. 2021

Distribución espacial de mosca mexicana de la fruta (Anastrepha spp.) (Diptera: Tephritidae) en Michoacán, México. Revista Colombiana de Entomología 47 (1): e7715. https://doi. org/10.25100/socolen.v47i1.7715

Recibido: 29-mar-2019

Aceptado: 27-ago-2020

Publicado: 29-abr-2021

Revista Colombiana de Entomología ISSN (Print): 0120-0488

ISSN (On Line): 2665-4385

https://revistacolombianaentomologia.univalle.edu.co

Open access

(c) (1) (2) (2) BY-NC-SA 4.0

Publishers: Sociedad Colombiana de Entomología SOCOLEN (Bogotá, D. C., Colombia) https://www.socolen.org.co

Universidad del Valle (Cali, Colombia)

https://www.univalle.edu.co

(C) 2021 Sociedad Colombiana de Entomología SOCOLEN y Universidad del Valle - Univalle
Resumen: A nivel global la fruticultura es una de las principales fuentes de ingreso, sin embargo, ésta es afectada por diversas plagas. En México una de las principales plagas que ocasiona un daño económico considerable es la mosca mexicana de la fruta, cuya ovipostura en la fruta causa que no sea aceptada en el mercado, con la consiguiente pérdida económica para los productores. Las alternativas de control contra dicha plaga han carecido de eficacia debido a que se desconoce su distribución espacial dentro de cultivos de ciruela, naranja, zapote, guayaba y mango. Por lo tanto, este trabajo tuvo por objetivo determinar la distribución espacial de las poblaciones de mosca mexicana de la fruta en dichos cultivos, mediante la aplicación de técnicas de estadística espacial que condujeron a la generación de mapas por medio del "krigeado". Los resultados demostraron que las poblaciones del insecto plaga presentan una distribución de tipo agregada, corroborada por los mapas de densidad. Las infestaciones se distribuyeron en diferentes áreas de los cultivos, lo cual puede resultar útil para dirigir las medidas de control sobre áreas específicas de infestación.

Palabras clave: Geoestadística, krigeado, mapas de densidad, distribución espacial, Anastrepha ludens, A. obliqua, A. serpentina, A. striata.

Abstract: Globally, fruit production is one of the main sources of income since it generates large economic spills for producers, however, it is affected by various pests. In Mexico, the Mexican fruit fly is one of the main pests that cause considerable economic damage. Fruit oviposited by this pest usually is no longer accepted in the market causing large economic losses to the producers. The alternative controls against this pest have been ineffective due, among other causes, to the fact that their spatial distribution within plum, orange, sapote, guava and mango orchards is unknown. Therefore, this work aimed to determine the spatial distribution of the Mexican fruit fly populations in these orchards, through the application of spatial statistics techniques and the generation of maps through "kriging". The results showed that populations of this fruit pest have an aggregate distribution, which was corroborated by density maps. Infestations were distributed in different areas of the orchards, which would be useful to direct control measures on specific areas of infestation.

Keywords: Geostatistics, krigging, density maps, spatial distribution, Anastrepha ludens, A. obliqua, A. serpentina, A. striata.

\section{Introducción}

México se ha consolidado como el quinto país productor a nivel mundial de naranja, con un volumen promedio de 4,2 millones de toneladas, las cuales se comercializaron tanto en el mercado nacional como internacional (Secretaría de Agricultura, Ganadería, Desarrollo Rural, Pesca y Alimentación 2017). Además, se considera el quinto país a nivel mundial como productor de mango, en donde destacan por su producción, los siguientes estados: Guerrero con 358 mil 235 toneladas, Sinaloa con 334 mil 239, Nayarit tiene 312 mil 39, Chiapas 237 mil 530, Oaxaca 158 mil 847 y Michoacán 146 mil 767 toneladas. Globalmente, es el quinto productor de 
guayaba, los meses de mayor disponibilidad de esta fruta son de octubre a noviembre, con aproximadamente el $41 \%$ de la producción nacional (Secretaría de Agricultura, Ganadería, Desarrollo Rural, Pesca y Alimentación 2017).

Las especies del género Anastrepha spp. están consideradas como las plagas principales en cultivos frutales al afectar directamente los frutos. El manejo de esta plaga se realiza con la aplicación de varias estrategias de control bajo un enfoque integral (Reyes et al. 2000). Estas plagas producen un daño directo cuando la hembra ovoposita en la pulpa de los frutos (Aluja 1994). Además, el daño provocado por la ovipostura favorece la entrada de hongos y bacterias que ocasionan la descomposición de la pulpa, aunado a las galerías de las larvas durante su alimentación. Todo esto produce una maduración precoz, caída del fruto y pérdida de cosecha (Curti-Díaz et al. 1998).

Las especies de Anastrepha de mayor importancia son: A. ludens (Loew, 1873), A. obliqua (Macquart, 1835), A. serpentina (Wiedemann, 1830) y A. striata (Schiner, 1868) de las cuales se desconoce su distribución espacial, necesaria para dirigir las medidas de control sobre áreas específicas de infestación. Por lo tanto, este trabajo tuvo por objetivo determinar la distribución espacial de las poblaciones de mosca mexicana de la fruta en cultivos de ciruela mexicana, naranja, zapote, guayaba y mango, mediante la aplicación de técnicas de estadística espacial que condujeron a la generación de mapas por medio del "krigeado".

\section{Materiales y métodos}

El estudio se llevó a cabo de marzo a septiembre del año 2018 en el oriente de Michoacán (México), principal zona productora de guayaba (Psidium guajava L.) y donde en las áreas marginales se encuentran otros hospederos como zapote (Diospyros digyna Jacq.), ciruela mexicana (Spondias purpurea L.), naranja (Citrus $\times$ sinensis Osbeck), mango (Mangifera indica L.). Cada uno de estos cultivos fueron muestreados. Esta región comprende los municipios de Zitácuaro, Benito Juárez, Jungapeo, Tuxpan y Ciudad Hidalgo. Para llevar a cabo el muestreo se colocaron 819 trampas tipo Jackson de manera aleatoria, cubriendo una superficie aproximada de 2.000 hectáreas, colocando una trampa en cada parcela experimental muestreada (819 en total), se realizó un solo muestreo utilizando como atrayente alcohol - metanol; las trampas utilizadas fueron facilitadas por la junta local de sanidad vegetal del oriente de Michoacán. La verificación de las trampas se realizó cada 15 días, hasta cubrir el área de estudio.

Análisis geoestadístico. Con el valor promedio de captura mensual de adultos con el programa variowin 2,2 (Software for spatial data analysis en 2D. New York, EE. UU.) se obtuvo el semivariograma experimental calculado con la siguiente expresión (Journel y Huijbregts 1978; Isaaks y Srivastava 1988):

$$
\gamma^{*}(h)=\frac{1}{2 N(h)} \sum_{i=1}^{N(h)}\left[z\left(x_{i}+h\right)-z\left(x_{i}\right)\right]^{2}
$$

donde: $\gamma^{*}(h)$ es el valor experimental del semivariograma para el intervalo de distancia $h ; N(h)$ es el número de pares de puntos muestrales separados por el intervalo de distancia $h ; z\left(x_{i}\right)$ es el valor de la variable de interés en el punto muestral $x_{i}$ y $z\left(x_{i}+h\right)$ es el valor de la variable de interés en el punto muestral $x_{i}+h$.

Estimación de los parámetros del modelo de semivariograma. Una vez obtenido el semivariograma experimental, se realizó su ajuste al semivariograma teórico (esférico, exponencial, gaussiano, etc.) Englund y Sparks (1988) utilizando el programa Variowin versión 2,2.

Validación del modelo teórico. Se siguió un procedimiento de validación cruzada, en el cual los parámetros del modelo Co (efecto de pepita), C + Co (meseta) y a (rango o alcance) fueron ajustados de forma interactiva (prueba y error) hasta obtener los valores de los estadísticos:

a. Media de los errores de estimación (MEE):

$$
\operatorname{MEE}=\frac{1}{n} \sum_{i=1}^{n}\left[z^{*}\left(x_{i}\right)-z\left(x_{i}\right)\right]
$$

dónde: $z^{*}\left(x_{i}\right)$ es el valor estimado de la variable de interés en el punto $x_{\mathrm{i}} ; \mathrm{z}\left(\mathrm{x}_{\mathrm{i}}\right)$ es el valor medido de la variable de interés en el punto $x_{i}$ y n es el número de puntos muestréales considerado en la interpolación.

El MEE no debe ser significativamente distinto de 0 (test t), en cuyo caso, indicaría que el modelo de semivariograma permite el cálculo de las estimaciones no sesgadas.

b. Error cuadrático medio (ECM):

$$
\mathbf{E C M}=\frac{1}{n} \sum_{i=1}^{n}\left[z^{*}\left(x_{i}\right)-z\left(x_{i}\right)\right]^{2}
$$

Un modelo de semivariograma se considera adecuado si, como regla práctica, el ECM es menor que la varianza de los valores muéstrales (Hevesi et al. 1992).

c. Error cuadrático medio adimensional (ECMA):

$$
\operatorname{ECMA}=\frac{1}{n} \sum_{i=1}^{n} \frac{\left[z^{*}\left(x_{i}\right)-z\left(x_{i}\right)\right]^{2}}{\sigma_{k}}
$$

donde: $\sigma_{k}$ es la desviación standar del error esperado en la estimación con el "krigeado".

La validez del modelo se satisface si ECMA está comprendido entre los valores $1 \pm 2(2 / \mathrm{N})^{0.5}$.

Nivel de dependencia espacial. La determinación del grado de relación entre los datos o nivel de dependencia espacial se obtuvo al dividir el efecto pepita entre la meseta expresando en el resultado en porcentaje. Si el resultado es menor de $25 \%$ el nivel de dependencia espacial es alta, si el rango está entre 26 y $75 \%$ el nivel es moderado y si es mayor del $76 \%$ el nivel de dependencia es bajo (Cambardella et al. 1994).

Elaboración de mapas. Para la estimación insesgada de valores asociados a puntos que no fueron muestreados se realizó la interpolación de valores a través del "krigeado" ordinario. Las estimaciones obtenidas fueron representadas en forma de mapa, mediante el uso del programa Surfer 9, Surface 266 Mapping System, (Golden Software Inc. 2021). 


\section{Resultados}

En las 2.000 ha muestreadas, la especie de mosca que registró mayor captura fue $A$. ludens seguida por $A$. striata y por $A$. obliqua. La distribución espacial que se obtuvo en las poblaciones de las tres especies de moscas fue de tipo agregada, los modelos a los que se adecuaron a los semivariogramas fueron esféricos y gaussianos (Tabla 1).

Para todos los modelos ajustados se observó un efecto pepita igual a cero, este hecho indicó que la escala de muestreo utilizada fue la correcta y el error de muestreo fue de 0 . El rango indica la distancia máxima hasta donde los datos muestreados tienen relación espacial entre sí, siendo en este caso bastante amplio. El valor de la meseta indica el valor asintótico de cada semivariograma. Todos los muestreos presentaron un alto nivel de dependencia espacial para cada uno de los mapas. Los modelos de la distribución espacial de las tres especies de moscas de la fruta fueron capturados y se validaron con los parámetros estadísticos al ubicarse dentro del rango permisible (Tabla 2 ).

En los mapas del área infestada (Fig. 1), en el caso del cultivo de ciruela se presentaron adultos de $A$. ludens y $A$. striata, siendo las poblaciones de la primera especie las de mayor abundancia. No hubo captura de A. obliqua en dicho cultivo. En los cultivos de guayaba se capturaron adultos de las tres especies, donde $A$. ludens y $A$. striata se situaron en la parte izquierda central del mapa y $A$. obliqua en el centro. En los cultivos de mango también se capturaron las tres especies pero con $A$. ludens la de mayor abundancia, ubicada en el centro del mapa con tendencia a crecer hacia el lado izquierdo; sin embargo, $A$. obliqua se situó en la parte inferior del mapa y $A$. striata se ubicó en el centro con tendencia a ser más abundante en la parte izquierda. En los cultivos de naranja $A$. ludens y $A$. obliqua se ubicaron en el centro del mapa, tendiendo a ser más abundantes en la parte superior derecha, mientras que $A$. striata se ubicó en el centro y en la parte inferior izquierda y derecha del mapa. Para el cultivo de zapote $A$. ludens se ubicó en la parte central del mapa, mientras que $A$. obliqua en la parte inferior izquierda, en tanto que $A$. striata se encuentra en la parte inferior derecha (Fig. 1).

En los cultivos se observó una infestación que fluctuó de $31 \%$ hasta el $88 \%$. Cabe destacar que en ciruela mexicana no se capturaron adultos de $A$. obliqua pero si en cultivos de guayaba en los que alcanzó el $88 \%$ de infestación (Tabla 3).

Tabla 1. Parámetros (efecto pepita, meseta y rango) de los modelos ajustados a los semivariogramas de las poblaciones de mosca de la fruta (Anastrepha spp.) en los cultivos de ciruela mexicana, guayaba, mango, naranja, zapote, en el año 2018. Meseta: valor asintótico de cada semivariograma. Rango: distancia máxima hasta donde los datos tienen relación espacial.

\begin{tabular}{|c|c|c|c|c|c|c|c|}
\hline Cultivo & Modelo & Pepita & Meseta & Rango & $\begin{array}{l}\text { Pepita/ } \\
\text { Meseta }\end{array}$ & $\begin{array}{c}\text { Nivel de } \\
\text { dependencia } \\
\text { espacial }\end{array}$ & $\begin{array}{l}\text { Coeficiente } \\
\text { de Curtosis }\end{array}$ \\
\hline \multicolumn{8}{|c|}{ Ciruela } \\
\hline Anastrepha ludens & Gaussiano & 0 & 9750 & 0,0228 & 0 & Alta & 1,21 \\
\hline Anastrepha striata & Esférico & 0 & 16000 & 0,0840 & 0 & Alta & 2,05 \\
\hline \multicolumn{8}{|c|}{ Guayaba } \\
\hline Anastrepha ludens & Esférico & 0 & 4554 & 0,2160 & 0 & Alta & 1,34 \\
\hline Anastrepha obliqua & Esférico & 0 & 4818 & 0,0299 & 0 & Alta & 1,29 \\
\hline Anastrepha. striata & Esférico & 0 & 5412 & 0,0616 & 0 & Alta & 2,46 \\
\hline \multicolumn{8}{|c|}{ Mango } \\
\hline Anastrepha ludens & Esférico & 0 & 27000 & 1,1250 & 0 & Alta & 2,05 \\
\hline Anastrepha obliqua & Esférico & 0 & 2160 & 0,0712 & 0 & Alta & 1,93 \\
\hline Anastrepha striata & Esférico & 0 & 11610 & 0,2799 & 0 & Alta & 1,03 \\
\hline \multicolumn{8}{|c|}{ Naranja } \\
\hline Anastrepha ludens & Gaussiano & 0 & 13749 & 0,2339 & 0 & Alta & 1,66 \\
\hline Anastrepha obliqua & Gaussiano & 0 & 10250 & 0,0280 & 0 & Alta & 2,24 \\
\hline Anastrepha striata & Gaussiano & 0 & 3213 & 0,0464 & 0 & Alta & 1,80 \\
\hline \multicolumn{8}{|c|}{ Zapote } \\
\hline Anastrepha ludens & Espacial & 0 & 10500 & 0,328 & 0 & Alta & 2,29 \\
\hline Anastrepha obliqua & Gaussiano & 0 & 15750 & 0,096 & 0 & Alta & 2,17 \\
\hline Anastrepha striata & Gaussiano & 0 & 14749 & 0,136 & 0 & Alta & 1,73 \\
\hline
\end{tabular}




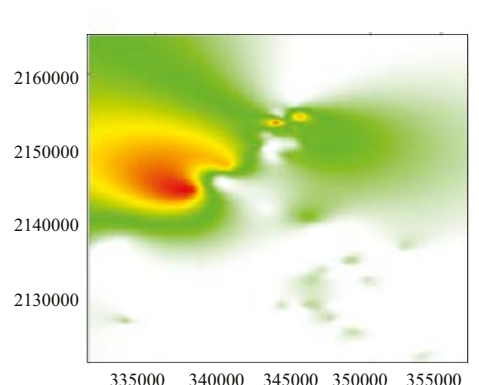

Anastrepha ludens en ciruela

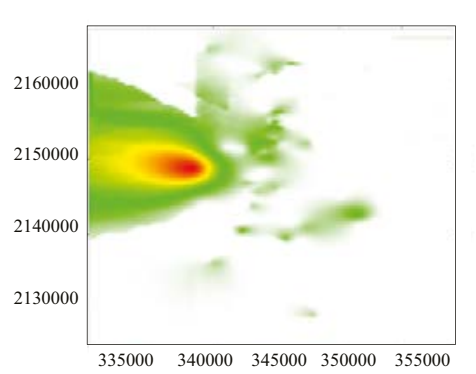

Anastrepha ludens en guayaba
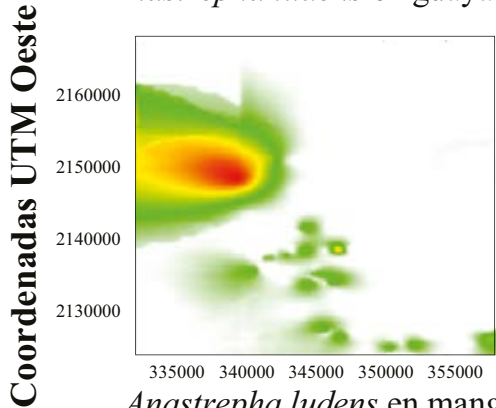

335000340000345000350000355000

Anastrepha ludens en mango

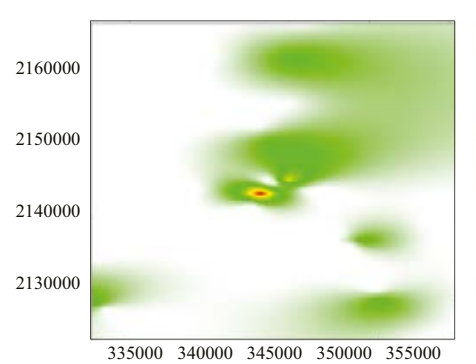

Anastrepha ludens en naranja

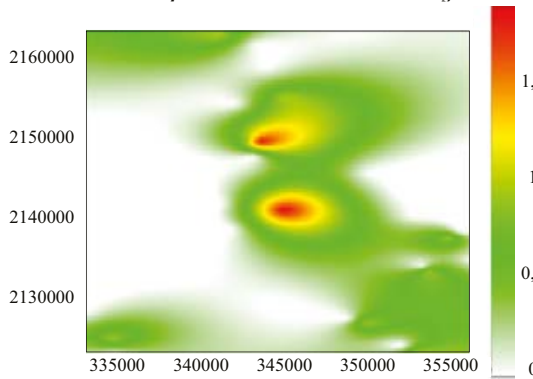

Anastrepha ludens en zapote

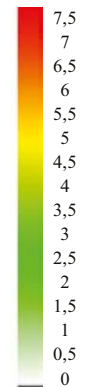

2160000
2150000
2140000
213000

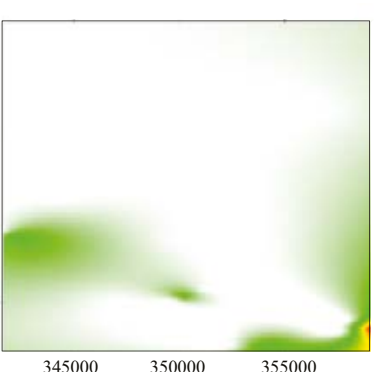

Anastrepha striata en ciruela

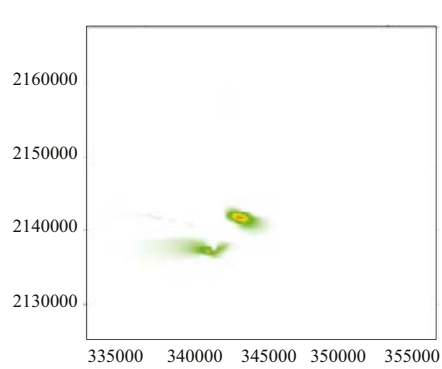

Anastrepha striata en guayaba

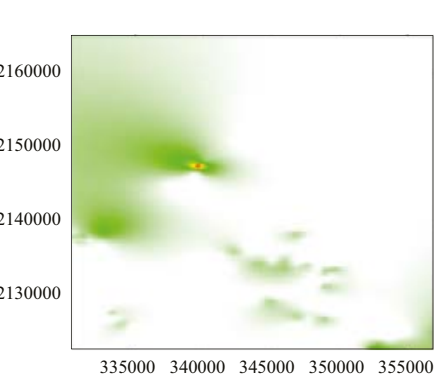

Anastrepha striata en mango
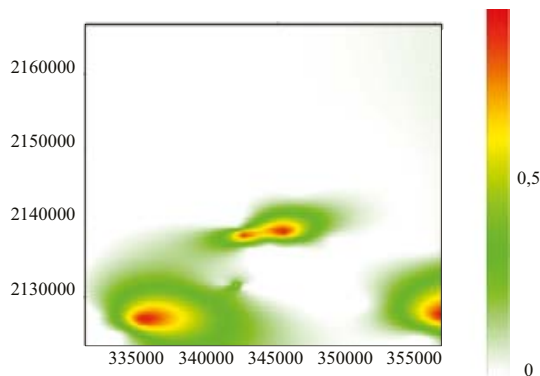

Anastrepha striata en naranja

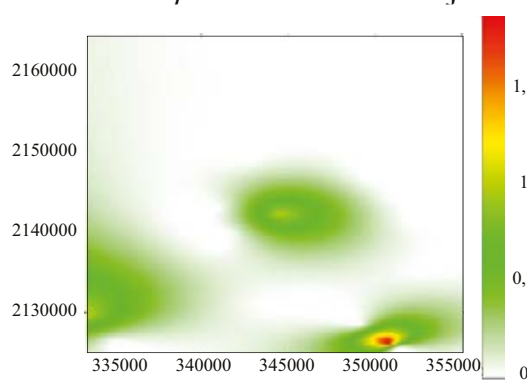

Anastrepha striata en zapote

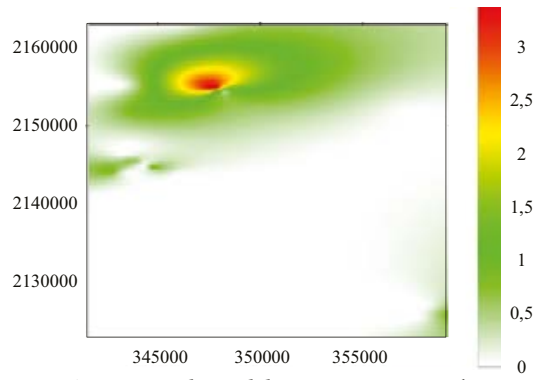

Anastrepha obliqua en guayaba
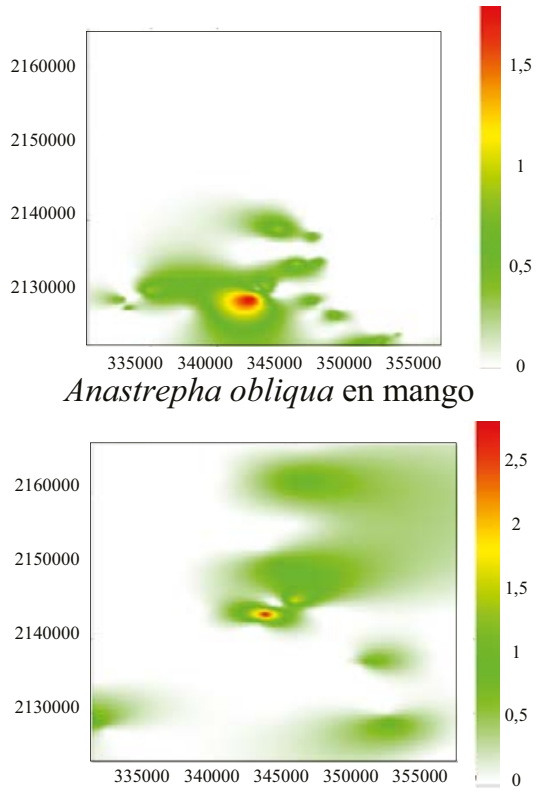

Anastrepha obliqua en naranja

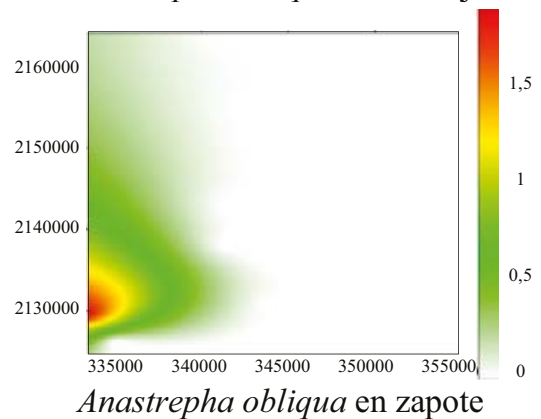

Coordenadas UTM Norte

Figura 1. Mapas de distribución espacial de las moscas de la fruta Anastrepha ludens, A. oblicua y A. striata, en cultivos de frutales en el oriente de Michoacán, México, 2018. 
Tabla 2. Valores de los estadísticos de la validación cruzada de los semivariogramas obtenidos en los muestreos de Anastrepha spp.: media de los errores de estimación (MEE), error cuadrado medio (ECM) y error cuadrático medio adimensional (ECMA).

\begin{tabular}{|c|c|c|c|c|c|c|c|}
\hline Cultivo & Muestreos & $\begin{array}{c}\text { Media } \\
\text { muestral }\end{array}$ & $\begin{array}{l}\text { Varianza } \\
\text { muestral }\end{array}$ & MEE* & $\begin{array}{l}\text { Varianza de } \\
\text { los errores }\end{array}$ & ECM & ECMA \\
\hline \multirow{2}{*}{ Ciruela } & Anastrepha ludens & 0,169 & 0,350 & $0,11^{\mathrm{ns}}$ & 0,123 & 0,10 & 1,13 \\
\hline & Anastrepha striata & 0,119 & 0,210 & $0,14^{\mathrm{ns}}$ & 0,156 & 0,12 & 1,10 \\
\hline \multirow{3}{*}{ Guayaba } & Anastrepha ludens & 0,162 & 0,304 & $0,10^{\text {ns }}$ & 0,219 & 0,14 & 1,06 \\
\hline & Anastrepha striata & 0,063 & 0,071 & $0,12^{\mathrm{ns}}$ & 0,030 & 0,11 & 1,11 \\
\hline & Anastrepha obliqua & 0,024 & 0,041 & $0,13^{\mathrm{ns}}$ & 0,026 & 0,09 & 1,09 \\
\hline \multirow{3}{*}{ Zapote } & Anastrepha ludens & 0,382 & 0,365 & $0,09^{\text {ns }}$ & 0,189 & 0,11 & 1,12 \\
\hline & Anastrepha striata & 0,114 & 0,163 & $0,10^{\mathrm{ns}}$ & 0,111 & 0,14 & 1,13 \\
\hline & Anastrepha obliqua & 0,059 & 0,118 & $0,14^{\mathrm{ns}}$ & 0,015 & 0,08 & 1,08 \\
\hline \multirow{3}{*}{ Naranja } & Anastrepha ludens & 0,161 & 0,269 & $0,08^{\mathrm{ns}}$ & 0,148 & 0,06 & 1,14 \\
\hline & Anastrepha striata & 0,081 & 0,075 & $0,10^{\mathrm{ns}}$ & 0,032 & 0,11 & 1,10 \\
\hline & Anastrepha obliqua & 0,048 & 0,047 & $0,07^{\mathrm{ns}}$ & 0,016 & 0,10 & 1,05 \\
\hline \multirow{3}{*}{ Mango } & Anastrepha ludens & 0,361 & 1,496 & $0,11^{\mathrm{ns}}$ & 1,105 & 0,13 & 1,12 \\
\hline & Anastrepha striata & 0,153 & 0,3565 & $0,13^{\mathrm{ns}}$ & 0,271 & 0,08 & 1,14 \\
\hline & Anastrepha obliqua & 0,076 & 0,080 & $0,09^{\mathrm{ns}}$ & 0,056 & 0,12 & 1,09 \\
\hline
\end{tabular}

\section{Discusión}

En la distribución de las especies de moscas de la fruta se encontró una mayor captura de $A$. ludens en comparación con A. obliqua y A. striata, y fue mayor en las zonas con temperaturas superiores a los $25^{\circ} \mathrm{C}$.

Las poblaciones de las tres especies de mosca de la fruta disminuyeron en la temporada de otoño-invierno y para los meses más cálidos (primavera - verano) esto está relacionado directamente con la temperatura que influye en su ciclo biológico (Comité Estatal de Sanidad Vegetal de Veracruz 2019).

Tabla 3. Superficie infestada de mosca de la fruta (Anastrepha spp.) dentro de las áreas de estudio, Michoacán, México en 2018.

\begin{tabular}{cc}
\hline Cultivo y especie de mosca & $\begin{array}{c}\text { \% de infestación en } \\
\text { los cultivos }\end{array}$ \\
\hline Ciruela, Anastrepha ludens & 42 \\
Ciruela, Anastrepha striata & 45 \\
Ciruela, Anastrepha obliqua & 0 \\
Guayaba, Anastrepha ludens & 38 \\
Guayaba, Anastrepha obliqua & 88 \\
Guayaba, Anastrepha striata & 46 \\
Mango, Anastrepha ludens & 48 \\
Mango, Anastrepha obliqua & 28 \\
Mango, Anastrepha strata & 36 \\
Naranja, Anastrepha ludens & 39 \\
Naranja, Anastrepha obliqua & 39 \\
Naranja, Anastrepha striata & 31 \\
Zapote, Anastrepha ludens & 50 \\
Zapote, Anastrepha obliqua & 28 \\
Zapote, Anastrepha striata & 35 \\
\hline
\end{tabular}

En el cultivo de ciruela no se encontró infestación de $A$. obliqua, contrario a lo reportado por Marín Patiño (2002) pero confirma que los frutos de $S$. mombin es un hospedante de A. obliqua.

La aplicación de las técnicas para modelizar la distribución espacial de insectos es una herramienta muy eficaz para determinar la distribución espacial de diversas especies de insectos plaga en diferentes cultivos. En estudios como el de Sciarretta et al. (2008) se ha reportado una distribución agregada con ajuste al modelo esférico en Lobesia botrana en plantaciones de uva. Así mismo Rivera-Martínez et al. (2017) determinaron la distribución espacial agregada en las poblaciones de adultos de Bactericera cockerelli Sulc., en el cultivo de tomate de cáscara (Physalis ixocarpa Brot.), También Jiménez et al. (2013) en poblaciones de trips en tomate de cáscara encontraron que exhiben una distribución agregada en varios centros de agregación y sus semivariogramas se ajustaron a los modelos esférico y gaussiano. Además, Bressan et al. (2010) determinaron la distribución espacial de Pentastiridius leporinus L., 1761, en el cultivo de trigo; Ramírez Dávila et al. (2011) mencionan que la distribución de Bactericera cockerelli en el cultivo de papa fue de tipo agregada.

Cuando los datos de la distribución espacial de un organismo son analizados con estadística clásica los resultados pueden variar, la diferencia está en que los modelos geoestadísticos proporcionan una medida más directa de la dependencia espacial, ya que consideran la naturaleza bidimensional de la distribución de los organismos a través de su exacta localización espacial y es independiente de la relación entre la media y la varianza (Samper y Carrera Ramírez 1996). Además, la geoestadística permite elaborar mapas de densidad de la distribución espacial de un organismo (Rossi et al. 1992; Ribes et al. 1998).

Moral García (2004) al utilizar la geoestadística para estudiar la distribución espacial del lepidóptero Helicoverpa armigera Hübner, en una parcela de tomate, con el empleo de la función variograma, obtuvo representaciones gráficas 
diarias de dichas distribuciones. Estos mapas se basan en la técnica de estimación geoestadística conocida como "krigeado", particularmente el ordinario en bloques. Contreras-Rendón et al. (2016) en un estudio de la distribución espacial de Candidatus Liberibacter solanacearum y de su vector Bactericera cockerelli Sulc en papa, obtuvieron mapas de densidad de $B$. cockerelli tanto en huevos, ninfas y adultos, usando el krigeado. En aguacate, Acosta-Guadarrama et al. (2017) mencionan el uso de mapas de distribución espacial de trips en diferentes épocas del año, para planear diversas estrategias de control (cultural, biológico o químico).

El comportamiento espacial de las poblaciones de las tres especies de mosca de la fruta de forma agregada en las parcelas de los diferentes frutales del oriente de Michoacán, permite sugerir que se pueden manejar $\mathrm{y}$, por lo tanto, reducirlas aplicando y dirigiendo medidas de control hacia los centros de agregación o puntos específicos en los que se encuentra la plaga, evitando aplicaciones generalizadas o de cobertura total, con un ahorro en los insumos, visualizando los focos de infestación a través de los mapas generados. Lo anterior concuerda con Tannure et al. (2013) quienes propusieron un manejo para controlar al pulgón del algodonero Aphis gossypii para las etapas temprana e intermedia del cultivo de algodón.

De acuerdo con los valores de cero que se registraron en el efecto pepita en todos los modelos ajustados, se puede afirmar que la escala de muestreo utilizada fue adecuada y el error de muestreo fue mínimo (Oliver y Webster 1991), esto sugiere que los modelos ajustados tienen un $98 \%$ de confiabilidad. Este hecho indicó que más del $90 \%$ de la variación total es debida a la dependencia espacial en la escala de muestreo utilizada; es decir, que más del $90 \%$ de la variación de la distribución de las poblaciones de mosca de la fruta se explica por la estructura espacial establecida con los semivariogramas (Liebhold y Sharov 1998). Valores de pepita iguales fueron obtenidos por Ramírez Dávila et al. (2011) en un estudio de la distribución espacial de Bactericera cockerelli en papa y por Solares Alonso et al. (2011) en la distribución espacial de trips en cultivos de aguacate.

El ajuste al modelo esférico indicó que dentro del área de muestreo hay puntos en los que la plaga tiene mayor abundancia con respecto a otros puntos, es decir que la infestación de la plaga que avanza a partir de una zona puntual, la infestación aumenta drásticamente en primavera. La distribución ajustada al modelo gaussiano reflejo que la agregación se expresó de forma continua dentro del área de muestreo. Estos puntos de agregación registraron un crecimiento rápido cerca de su origen pero decrecen conforme aumenta la distancia, esto es factible dado el comportamiento de las especies de mosca de la fruta, los focos de infestación se observan en los mapas obtenidos. Moral García (2006) al realizar la distribución espacial de $H$. armigera y sus variogramas teóricos, encontró que se ajustaron a modelos esféricos o exponenciales. Contreras-Rendón et al. (2016) encontraron que los semivariogramas para huevos y ninfas de $B$. cockerelli se ajustaron al modelo esférico, lo que indicó la agregación de la plaga en ciertas zonas de la parcela.

El alto nivel de dependencia espacial que resultó de dividir el efecto pepita entre la meseta fue menor a $25 \%$ para todos los semivariogramas con una alta dependencia espacial. Esto sugiere que las poblaciones de moscas de la fruta dependen entre sí y que el nivel de agregación es alto (Rossi et al. 1992). Esquivel Higuera y Jasso García (2014) en un estudio de la distribución espacial de Spodoptera exigua demostraron que hay una estructura espacial agregada, con un nivel de dependencia espacial alto para las seis localidades en estudio.

En los mapas de densidad que fueron generados se observan los centros de agregación que presentó el insecto dentro de la parcela, lo que indica que el control de la plaga se puede hacer con mayor precisión (Weisz et al. 1996; Ribes et al. 1998; Moral García 2004; Rong et al. 2006; Esquivel Higuera y Jasso García 2014). Si se mejoran las estrategias de control también se reduce la presión de selección y se puede retrasar el desarrollo de resistencia en los insectos a los plaguicidas debido a la creación de refugios temporales dinámicos, al no tratar toda la superficie del cultivo (Moral et al. 2011). Con estas acciones se evitará una mayor distribución de las tres especies de mosca de la fruta y que causen mayores daños económicos.

Considerando la estructura de los mapas, las áreas de infestación de las especies de mosca de la fruta que se distribuyeron de un $31 \%$ a un $88 \%$ de la parcela tienen similitud a lo reportado por Esquivel Higuera y Jasso García (2014) para la distribución espacial de $S$. exigua en maíz con un porcentaje máximo de $94 \%$. Así mismo, Jiménez et al. (2013) y Arce-Flores et al. (2014) reportaron que las poblaciones de Frankliniella occidentalis no se distribuyen en la totalidad de las parcelas en tomate de cáscara y de nardo, respectivamente. Todo lo anterior confirma que la infestación de los insectos no se presenta uniforme dentro de los cultivos.

Con el uso de los mapas generados en este estudio se pueden sugerir acciones a los productores para llevar a cabo acciones que permitan reducir las poblaciones de los insectos plaga. Entre los métodos a sugerir están, el control biológico en todas sus modalidades, el control cultural, el control físico, el control químico, entre otros. La implementación de los métodos geoestadísticos en el manejo integrado de esta plaga puede ser de gran impacto para los productores, ya que permite conocer con mayor precisión y eficiencia el comportamiento espacial de este insecto. Los mapas elaborados mediante estos métodos de estadística espacial proporcionarán a las autoridades competentes y al personal especializado, un componente novedoso y de gran ayuda para el desarrollo de nuevos y más pertinentes programas de manejo integrado de este problema fitosanitario, que sin duda alguna, impactará positivamente en la producción frutícola de la región.

\section{Literatura citada}

ACOSTA-GUADARRAMA, A. D.; RAMÍREZ-DÁVILA, J. F.; RIVERA-MARTÍNEZ， R.; FIGUEROA-FIGUEROA， D. K.; LARA-DIAZ, A. V.; MALDONADO-ZAMORA, F. I.; TAPIA-RODRÍGUEZ, A. 2017. Distribución espacial de Trips spp. (Thysanoptera) y evaluación de su control mediante el depredador Amblyseius swirskii en el cultivo de aguacate en México. Southwestern Entomologist 42 (2): 435-446. https:// doi.org/10.3958/059.042.0214

ALUJA, M. 1994. Bionomics and management of Anastrepha. Annual Review Entomology 39: 155-178. https://doi.org/10.1146/ annurev.en.39.010194.001103

ARCE-FLORES, J.; LÓPEZ-MARTÍNEZ, V.; GAÓNA-GARCÍA, Á. 2014. Fluctuación poblacional y distribución de Frankliniella occidentalis (Pergande) (Thysanoptera: Thripidae) en nardo en 
Morelos, México. Acta Agrícola y Pecuaria 1 (1): 37-42. https:// dialnet.unirioja.es $/$ servlet $/$ articulo? codigo $=6201339$

BRESSAN, A.; MORAL GARCÍA, F. J.; SÉMÉTEY, O.; BOUDON-PADIEU, E. 2010. Spatio-temporal pattern of Pentastiridiusleporinus migration in an ephemeral cropping system. Agricultural and Forest Entomology 12 (1): 59-68. https://doi.org/10.1111/j.1461-9563.2009.00450.x

CAMBARDELLA, C. A.; MOORMAN, T. B.; NOVAK, J. M.; PARKIN, T. B.; KARLEN, D. L.; TURCO, R. F.; KONOPKA, A. E. 1994. Field-scale variability of soil properties in central Iowa soils. Soil Science Society of America Journal 58 (5): 1501-1511. https://doi.org/10.2136/sssaj1994.03615995005800050033x

COMITÉ ESTATAL DE SANIDAD VEGETAL DE VERACRUZ. 2019. México. CESVVER. Disponible en: http://www.siap.sagarpa.gob.mx [Fecha revisión: 23 marzo 2017].

CONTRERAS-RENDÓN, A.; GUTIÉRREZ-IBÁÑEZ, A. T.; SILVA-ROJAS, H. V.; SÁNCHEZ-PALE, J. R.; LAGUNA-CERDA, A.; RAMÍREZ-DÁVILA, J. F. 2016. Distribución espacial de Candidatus Liberibacter solanacearum y Bactericera cockerelli (Sulc) (Hemiptera: Triozidae) en papa (Solanum tuberosum L.). Southwestern Entomologist 41 (1): 105-114. https://doi.org/10.3958/059.041.0112

CURTI-DÍAZ, S.; DÍAZ-ZORRILLA, U.; LOREDO-SALAZAR, X.; SANDOVAL RINCÓN, J. A.; PASTRANA APONTE, L.; RODRÍGUEZ CUEVAS, M. 1998. Manual de producción de naranja para Veracruz y Tabasco. Libro Técnico $\mathrm{N}^{\circ} 2$. Instituto Nacional de Investigaciones Forestales, Agrícolas y Pecuarias. INIFAP - CIRGOC - SAGAR. México. 175 p. http://www.concitver.com/archivosenpdf/MANUAL $\% 20$ DE\%20PRODUCCION\%20DE\%20NARANJA\%20PARA\%20 VERACRUZ\%20Y\%20TABASCO.pdf

ENGLUND, E. J.; SPARKS, A. R. 1988. GEO-EAS (Geostatistical Environmental Assessment Software) User's guide. Environmental protection agency document EPA/600/4-88/033. Environmental Monitoring Systems Laboratory, EE. UU. https:// www.osti.gov/biblio/6077300

ESQUIVEL HIGUERA, V.; JASSO GARCÍA, Y. 2014. Distribución espacial y mapeo de gusano soldado en seis localidades del Estado de México, en el año 2011. Revista Mexicana de Ciencias Agrícolas 5: 923-935. http://www.scielo.org.mx/scielo. php?script=sci_arttext\&pid=S2007-09342014000600002

GOLDEN SOFTWARE. 2021. Surfer 9, Surfer explore the depths of your data. https://www.goldensoftware.com/products/surfer

HEVESI, J. A.; ISTOK, J. D.; FLINT, A. L. 1992. Precipitation estimation in mountainous terrain using multivariate geostatistics. Part. I. Structural analysis. Journal of Applied Meteorology 31 (7): 661-676. https://doi.org/10.1175/1520-0450(1992)031<0661:PEIMTU $>2.0 . \mathrm{CO} ; 2$

ISAAKS, E. H.; SRIVASTAVA, R. M. 1988. Spatial continuity measures for probabilistic and deterministic geostatistics. Mathematical Geology 20 (4): 313-341. https://doi.org/10.1007/ BF00892982

JIMÉNEZ C., R. Á.; RAMÍREZ D., J. F.; SÁNCHEZ P., J. R.; SALGADO S., M. L.; LAGUNA C., A. 2013. Modelización espacial de Frankliniella occidentalis (Thysanoptera: Thripidae) en tomate de cáscara por medio de técnicas geoestadísticas. Revista Colombiana de Entomología 39 (2): 1-10. http://www.scielo.org. co/pdf/rcen/v39n2/v39n2a01.pdf

JOURNEL, A. G.; HUIJBREGTS, C. J. 1978. Mining geostatistics. Academic Press, Londres, Reino Unido. 600 p.

LIEBHOLD, A. M.; SHAROV, A. A. 1998. Testing for correlation in the presence of spatial autocorrelation in insect count data. pp. 111-117. En: Baumgartner, J.; Brandmayr, P.; Manly, B. F. J. (Eds.). Population and community ecology for insect management and conservation. Balkema, Rotterdam, Netherlands. https://doi.org/10.1201/9780429333422-10

MARÍN PATIÑO, M. L. 2002. Identificación y caracterización de moscas de las frutas en los departamentos del Valle del Cauca, Tolima y Quindío. Proyecto de Entomología. Universidad de
Caldas Facultad de Ciencias Agropecuarias. Programa de Agronomía. Manizales, Colombia. 29 p. http://ciat-library.ciat.cgiar. org/Articulos_Ciat/mosca_fruta.pdf

MORAL, F. J.; TERRÓN, J. M.; REBOLLO, F. J. 2011. Site-specific management zones based on the Rasch model and geostatistical techniques. Computers and Electronic in Agriculture 75 (2): 223-230. https://doi.org/10.1016/j.compag.2010.10.014

MORAL GARCÍA, F. J. 2004. Aplicación de la geoestadística en las ciencias ambientales. Revista Ecosistemas 13 (1): 78-86. https:// www.revistaecosistemas.net/index.php/ecosistemas/article/ view/582

MORAL GARCÍA, F. J. 2006. Analysis of the spatio-temporal distribution of Helicoverpa armigera $\mathrm{Hb}$. in a tomato field using a stochastic approach. Biosystems Engineering 93 (3): 253-259. https://www.sciencedirect.com/science/article/abs/pii/ S1537511005003016?via\%3Dihub

OLIVER, M. A.; WEBSTER, R. 1991. How geostatistics can help you. Soil Use and Management 7 (4): 206-217. https://doi. org/10.1111/j.1475-2743.1991.tb00876.x

RAMÍREZ DÁVILA, J. F.; PORCAYO CAMARGO, E.; SÁNCHEZ PALE, J. R. 2011. Análisis de la distribución espacial de Bactericera cockerelli Sulc (Hemiptera: Triozidae) en Solanum tuberosum L. en Donato Guerra, México. Boletín del Museo de Entomología de la Universidad del Valle 12 (1): 12-24. https://1library.co/document/yj724n2y-analisis-distribucionespacial-bactericera-cockerelli-hemiptera-triozidae-tuberosum. html\#pdf-content

REYES, J.; SANTIAGO, G.; HERNÁNDEZ, P. 2000. Mexican fruit fly eradication programme. pp. 337-380. En: Tan, K.-H. (Eds.). Area wide control of fruits flies and other insect pest. Penerbit Universiti Sains Malaysia, Penang, Malaysia. ISBN 983-861195-6

RIBES, M.; BASCUÑANA, M.; AVILLA, J. 1998. Estudio de la distribución espacial de Cydia pomonella (L.) y Pandemis heparana (Denis \& Schiffermüller) en Torregrossa (Lleida) mediante métodos geoestadísticos. Boletín de Sanidad Vegetal Plagas 24 (4): $935-$ 948. https://repositori.udl.cat/bitstream/handle/10459.1/41623/ pdf_plagas_BSVP-24-04-Adenda-0935-0948.pdf;jsessionid= C0233A045508129B54DFDF90E11A8A13? sequence $=1$

RIVERA-MARTÍNEZ, R.; ACOSTA-GUADARRAMA, A. D.; RAMÍREZ-DÁVILA, J. F.; FIGUEROA-FIGUEROA, D. K.; MALDONADO-ZAMORA, F. I.; LARA-DÍAS, A. V. 2017. Distribución Espacial de las Poblaciones de Adultos de Bactericera cockerelli Sulc. en el cultivo de Tomate de Cáscara (Physalis ixocarpa Brot.). Southwestern Entomologist 42 (4): 1057-1068. https://doi.org/10.3958/059.042.0424

RONG, J.; DIAN-MO, L.; BAO-YU, X.; ZHE, L.; DONG-LI, M. 2006. Spatial distribution of oriental migratory locust (Orthoptera: Acrididae) egg pod populations: Implications for site-specific pest management. Environmental Entomology 35 (5): 1244-1248. https://doi.org/10.1093/ee/35.5.1244

ROSSI, R. E.; MULLA, J. D.; JOURNEL, A. G.; FRANZ, E. H. 1992. Geostatical tools for modeling and interpreting ecological spatial dependence. Ecological Monographs 62 (2): 277-314. https://doi.org/10.2307/2937096

SAMPER, F. J.; CARRERA RAMÍREZ, J. 1996. Geoestadística, aplicaciones a la hidrogeología subterránea. 2 da. Edición. Centro Internacional de Métodos Numéricos en Ingeniería, Barcelona, España. 484 p. 
SCIARRETTA, A.; ZINNI, A.; MAZZOCCHETTI, A.; TREMATERRA, P. 2008. Spatial analysis of Lobesia botrana (Lepidoptera: Tortricidae) male population in a Mediterranean agricultural landscape in central Italy. Environmental Entomology 37 (2): 382-390. https://doi.org/10.1093/ee/37.2.382

SECRETARÍA DE AGRICULTURA, GANADERÍA, DESARROLLO RURAL, PESCA Y ALIMENTACIÓN. 2017. México. SAGARPA. Disponible en: http://www.siap.sagarpa.gob.mx/ [Fecha de revisión: 23 marzo 2017].

SOLARES ALONSO, V. M.; RAMÍREZ DÁVILA, J. F.; SÁNCHEZ PALE, J. R. 2011. Distribución espacial de trips (Insecta: Thysanoptera) en el cultivo de aguacate (Persea americana Mill.). Boletín del Museo de Entomología de la Universidad del Valle 12 (2): 1-12. https://core.ac.uk/reader/11863710

TANNURE, C. L.; MAZZA, S. M.; GIMÉNEZ, L. I. 2013. Modelos para caracterizar los patrones de distribución espacial de Aphis gossypii (Homoptera: Aphididae), en el cultivo de algodón (Gossypium hirsutum). Revista Facultad de Ciencias Agrarias, UNNE. 1-4. https://www.academia.edu/6199666/Modelos para_caracterizar_los_patrones_de_distribuci\%C3\%B3n espacial_de_Aphis_gossypii_Homoptera_Aphididae_en_el_ cultivo_de_algod $\% \bar{C} 3 \%$ B3n_Gossypium_hirsutum

WEISZ, R.; FLEISCHER, S.; SMILOWITZ, Z. 1996. Site specific integrated pest management for high value crops: Impact on potato pest management. Journal of Economic Entomology 89 (2): 501-509. https://doi.org/10.1093/jee/89.2.501

\section{Origen y financiación}

El origen del artículo es un trabajo realizado como Tesis para obtener el grado de Ingeniero Agrónomo Fitotecnista por la Facultad de Ciencias Agrícolas de la Universidad Autónoma del Estado de México, dicho trabajo fue realizado por el alumno Ricardo Vanegas Carrillo, quien funge como primer autor del artículo de investigación. El financiamiento para el trabajo fue proporcionado en parte por el Comité Estatal de Sanidad Vegetal del Estado de Michoacán, México, dicha Institución le proporcionó al alumno viáticos para gasolina y alimentación. La otra parte del financiamiento fue proporcionado por el Laboratorio de Investigaciones Entomológicas y Tecnologías en Agricultura de Precisión de la Facultad de Ciencias Agrícolas de la UAEMex, se le proporcionó al alumno el material necesario para realizar el trampeo y el análisis de los resultados.

\section{Contribución de los autores}

Ricardo Vanegas-Carrillo realizó los muestreos respectivos durante todo el trabajo de investigación y formó parte del análisis que se llevó a cabo con los datos resultantes. Dr. José Francisco Ramírez-Dávila participó en el análisis de los resultados en todas sus etapas. Dr. Roberto Rivera-Martínez participó en parte del proceso de análisis de los resultados obtenidos. 